\title{
ABCG2 Deficiency Does Not Alter Dolutegravir Metabolism and Pharmacokinetics
}

\author{
Junjie Zhu, Xin Tian, Amina I. Shehu, Deborah K. McMahon, and Xiaochao Ma \\ Center for Pharmacogenetics, Department of Pharmaceutical Sciences, School of Pharmacy (J.Z., X.T., A.I.S., X.M.) and Division \\ of Infectious Disease, Department of Medicine (D.K.M.), University of Pittsburgh, Pittsburgh, Pennsylvania
}

Received December 4, 2019; accepted April 10, 2020

\section{ABSTRACT}

Dolutegravir (DTG) is a potent integrase inhibitor of human immunodeficiency virus. Because DTG is a substrate of the efflux transporter ABCG2 and ABCG2 is highly polymorphic, we asked whether dose adjustment of DTG is needed for ABCG2-deficient individuals. Using Abcg2-null mice, the current work investigated the impact of ABCG2 deficiency on DTG metabolism and pharmacokinetics. Compared with wild-type mice, no statistically significant difference was found in the systemic and tissue-specific (liver, kidney, and brain) pharmacokinetics of DTG in Abcg2-null mice. In addition, ABCG2 deficiency had no statistically significant impact on the production and excretion of DTG metabolites. In summary, this study demonstrated that deficiency of ABCG2 does not alter DTG metabolism and pharmacokinetics, suggesting that dose adjustment of DTG is not needed for individuals with ABCG2 deficiency.

\section{SIGNIFICANCE STATEMENT}

The current work demonstrated that deficiency of ATP-binding cassette subfamily G member 2 (ABCG2) does not alter Dolutegravir (DTG) metabolism and pharmacokinetics, suggesting that dose adjustment of DTG is not needed for individuals with ABCG2 deficiency.

\section{Introduction}

Dolutegravir (DTG) is an integrase inhibitor of human immunodeficiency virus (HIV) with potent antiretroviral activity (Cahn et al., 2013; Raffi et al., 2013). DTG overcomes HIV drug resistance to the first generation of integrase inhibitors (Hightower et al., 2011; Eron et al., 2013). In addition, DTG inhibits the replication of viral strains resistant to other marketed anti-HIV drugs, including nucleoside and non-nucleoside reverse transcriptase inhibitors (Kobayashi et al., 2011). Therefore, DTG-containing regimens are highly recommended for antiretroviral therapy (Phillips et al., 2018).

DTG is rapidly absorbed after oral administration (Min et al., 2010). The predominant circulating component in plasma is DTG, whereas major metabolites found in urine and feces are glucuronide M2, cysteine adduct M4, and the hydroxylated metabolite M3 and its hydrolytic products M1 and M5 (Castellino et al., 2013; Zhu et al., 2018a) (Fig. 1). Among these metabolites, M2 and M3 are produced by UDP-glucuronosyltransferase (UGT) $1 \mathrm{~A} 1$ and CYP3A4, respectively (Reese et al., 2013). The identification of M4 indicates the production of a reactive intermediate that interacts with glutathione. In addition to the phase I and II

This work was supported in part by the National Institutes of Health National Institute of Allergy and Infectious Diseases [Grant R01AI131983]. https://doi.org/10.1124/jpet.119.264424. metabolism of DTG, a cell-based assay suggests that DTG is a substrate of ATP-binding cassette subfamily B member 1 (ABCB1) and ATP-binding cassette subfamily G member 2 (ABCG2) (Fig. 1) (Reese et al., 2013).

ABCG2 is an efflux transporter expressed in multiple organs, including the liver, kidney, and brain (Maliepaard et al., 2001; Fetsch et al., 2006). In the liver, ABCG2 is expressed on the bile canalicular membrane of hepatocytes and plays a vital role in biliary excretion of a broad range of drugs (Maliepaard et al., 2001; Fetsch et al., 2006). In the brain, ABCG2 is one of the primary components in bloodbrain barrier extruding xenobiotics and endobiotics from the brain (Eisenblätter et al., 2003; Nicolazzo and Katneni, 2009). Therefore, blockage and/or deficiency of ABCG2 can increase the hepatic, cerebral, and systemic drug exposure and potentially lead to adverse drug reactions (Hirano et al., 2005). For instance, a high exposure of rosuvastatin and atorvastatin has been observed in individuals with ABCG2 deficiency (Keskitalo et al., 2009; Wan et al., 2015). Because ABCG2 is important in modulating drug disposition, dose adjustment should be considered for ABCG2-deficient individuals receiving drugs as ABCG2 substrates (Hira and Terada, 2018).

ABCG2 deficiency is usually caused by loss-of-function mutations of ABCG2. Forty-three single nucleotide polymorphisms (SNP) have been identified in genetic analysis of ABCG2 (Iida et al., 2002). SNP rs2231142 resulted in a replacement

ABBREVIATIONS: ABCB1, ATP-binding cassette subfamily B member 1; ABCG2, ATP-binding cassette subfamily G member 2; AUC, area under concentration-time curve; DTG, dolutegravir; HIV, human immunodeficiency virus; PCR, polymerase chain reaction; p.o., per os; PPIX, protoporphyrin IX; QTOFMS, quadrupole time-of-flight mass spectrometry; SNP, single nucleotide polymorphism; UGT, UDP-glucuronosyltransferase; UPLC, ultra-performance liquid chromatography; WT, wild-type. 


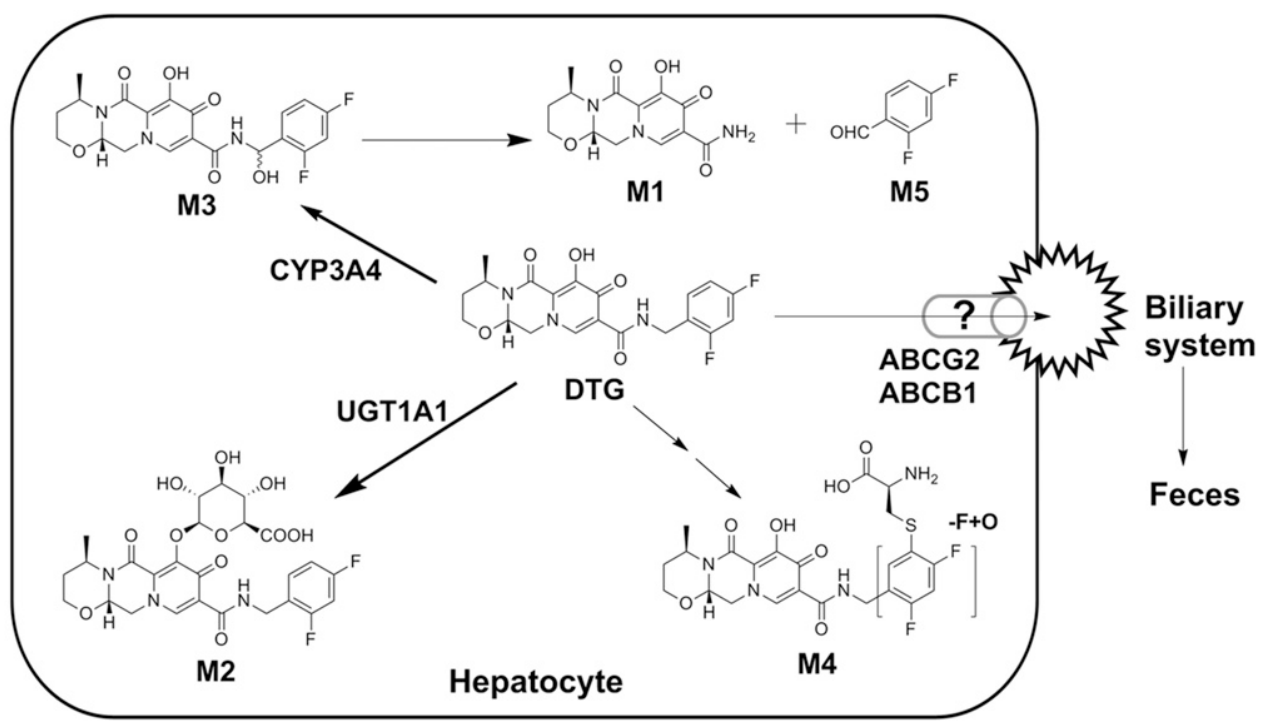

Fig. 1. DTG metabolism and disposition in hepatocytes. M1, M2, M3, M4, and M5 are the metabolites of DTG. M2 and M3 are formed by UGT1A1 and CYP3A4, respectively. $\mathrm{ABCG} 2$ and $\mathrm{ABCB} 1$ are the potential transporters mediating the efflux of DTG from hepatocytes to the biliary system. The current work investigated the impact of ABCG2 deficiency on DTG metabolism and pharmacokinetics in mice.

of glutamine by lysine, leading to a lower expression level of ABCG2 and influencing the clinical outcomes of drugs that are ABCG2 substrates (Imai et al., 2002; Hira and Terada, 2018). Although cell-based assays revealed ABCB1 and ABCG2 as transporters of DTG (Reese et al., 2013), high plasma DTG concentrations were only observed in individuals with ABCG2 SNP rs2231142 (Tsuchiya et al., 2017). Neurotoxicity is one of the major adverse effects of DTG (de Boer et al., 2016; Elzi et al., 2017), which leads to DTG discontinuation in $\sim 6 \%$ patients (Hoffmann et al., 2017). Although the mechanism of DTG neurotoxicity remains unclear, high plasma DTG concentrations are associated with DTG neurotoxicity (Yagura et al., 2017; Parant et al., 2018). All these data raised the concern whether dose adjustment of DTG is needed in ABCG2-deficient individuals.

The current study aimed to investigate the impact of ABCG2 deficiency on DTG metabolism and disposition (Fig. 1). Abcg2-null mice were used to mimic the ABCG2deficient condition in humans. Systemic and tissue-specific (liver, kidney, and brain) pharmacokinetics of DTG were compared between wild-type (WT) and Abcg2-null mice. In addition, the excretions of DTG and its metabolites were investigated in the urine and feces of WT and Abcg2-null mice.

\section{Materials and Methods}

Chemicals and Reagents. DTG and protoporphyrin IX (PPIX) were purchased from Sigma-Aldrich (St. Louis, MO). Solvents for metabolite analysis were purchased from Fisher Chemical (Fair Lawn, NJ).

Animals and Treatments. Abcg2-null mice were originally generated in Dr. Schinkel's group (Jonker et al., 2002) and purchased from Taconic Biosciences (Hudson, NY). Abcg2-null mice were verified by polymerase chain reaction (PCR) genotyping. The primers used for genotyping were as follows: 5' -CTTCTCCATTCATCAGCCTCG-3'; $5^{\prime}$ CAGTCGATGGATCCACTTAGG-3'; and 5'-GGAGCAAAGCTGCTA TTGGC- $3^{\prime}$. A PCR band at $368 \mathrm{bp}$ indicated the WT allele, whereas the ABCG2-null allele was evident with a 594-bp band. To validate ABCG2 function in Abcg2-null mice, PPIX, an endogenous substrate of ABCG2, was measured in liver samples collected from WT and Abcg2-null mice (male, 7-10 weeks, $n=4$ for each group) without any treatment. For the pharmacokinetic study, WT and Abcg2-null mice (male, 7-10 weeks, $n=4$ for each group) were treated with DTG (20 mg/kg, p.o.). Blood, liver, kidney, and brain were collected at different time points ( $0-48$ hours) after DTG treatment. Another two groups of WT and Abcg2-null mice ( $n=4$ for each group) were treated with DTG (20 mg/kg, per os (p.o.)) and housed separately in metabolic cages overnight to collect urine and feces. All animal studies were approved by the Institutional Animal Care and Use Committee of the University of Pittsburgh.

Sample Preparation for Metabolite Analysis. The extraction of DTG and its metabolites from sera, tissues, urines, and feces was performed according to previous reports with minor modification (Bennetto-Hood et al., 2014; Liu et al., 2017; Zhu et al., 2018b). For serum, $15 \mu \mathrm{l}$ of serum sample was mixed with $90 \mu \mathrm{l}$ of acetonitrile followed by vortexing and centrifugation to precipitate the protein. For the liver, $100 \mathrm{mg}$ of liver sample was homogenized in $600 \mu \mathrm{l}$ of watermethanol $(1: 2, \mathrm{v} / \mathrm{v})$, and $600 \mu \mathrm{l}$ of acetonitrile was added to the resulting suspension. Then, the mixture was vortexed for 2 minutes followed by centrifugation at $15,000 \mathrm{rpm}$ for 10 minutes. For the urine, $100 \mu \mathrm{l}$ of urine was mixed with $400 \mu \mathrm{l}$ acetonitrile followed by centrifugation at $15,000 \mathrm{rpm}$ for 10 minutes. Feces were homogenized in water $(50 \mathrm{mg}$ of feces in $500 \mu \mathrm{l}$ of water). Four hundred microliters of acetonitrile was added to $200 \mu \mathrm{l}$ of the resulting suspension and vortexed for 1 minute. Subsequently, the resulting mixture was centrifuged at 15,000 rpm for 10 minutes. PPIX in mouse liver was extracted according to a previous report (Wang et al., 2019). Briefly, $50 \mathrm{mg}$ of liver sample was homogenized in $250 \mu \mathrm{l}$ of water, and the resulting $100 \mu \mathrm{l}$ of suspension was mixed with $200 \mu \mathrm{l}$ of methanolacetonitrile $(1: 1, \mathrm{v} / \mathrm{v})$ followed by centrifugation at $15,000 \mathrm{rpm}$ for 10 minutes. Metabolites in the final supernatants were analyzed by ultra-performance liquid chromatography (UPLC) and quadrupole time-of-flight mass spectrometry (QTOFMS) (Waters Corporation, Milford, MA).

UPLC-QTOFMS Analysis and Pharmacokinetic Analysis. The analyses of DTG and its metabolites were performed on an Acquity UPLC BEH C18 column $(2.1 \times 100 \mathrm{~mm}, 1.7 \mu \mathrm{m})$ coupled with a SYNAPT G2-S mass spectrometer (Waters Corporation). Column temperature was maintained at $50^{\circ} \mathrm{C}$. The mobile phases for metabolite analysis were A $(0.1 \%$ formic acid in water) and B $(0.1 \%$ formic acid in acetonitrile) and were delivered at a flow rate of $0.5 \mathrm{ml} / \mathrm{min}$ with a gradient elution $(0.0-1.0$ minute, $2 \% \mathrm{~B} ; 1.0-12.0$ minutes, $2 \%-95 \% \mathrm{~B} ; 12.0-20.0$ minutes, $95 \% \mathrm{~B} ; 20.0-20.5$ minutes, $95 \%-2 \% \mathrm{~B}$; 20.5-24 minutes, $2 \%$ B). PPIX assay was conducted using a previously reported method (Wang et al., 2019). In brief, PPIX analysis was performed on an Acquity UPLC BEH C18 column $(2.1 \times 50 \mathrm{~mm}$, $1.7 \mu \mathrm{m})$, and acetonitrile-water containing $2 \mathrm{mM}$ of ammonium 
bicarbonate and $0.05 \%$ aqueous ammonia was used as the mobile phase. The QTOFMS system was operated in both positive and negative modes with electrospray ionization. The detailed mass parameters were the same as previously reported (Zhu et al., $2018 b$ ). Collision energy ramping from 15 to $45 \mathrm{eV}$ was used for structural elucidations of DTG metabolites. DTG levels in serum, liver, kidney, brain, feces, and urine were determined by the calibration curve made by different concentrations of DTG standard spiked in the corresponding blank tissue matrix. The quantification method of DTG was validated by accuracy, linearity, and recovery. DTG metabolites were identified based on accurate mass measurement (mass errors less than $10 \mathrm{ppm}$ ) and MS/MS fragmental analysis, and they were relatively qualified using their peak areas of the mass signals with MassLynx (Milford, MA). Systemic pharmacokinetic parameters were calculated by noncompartmental model analysis on Phoenix WinNonlin. Tissue pharmacokinetic parameters were calculated by Graphpad Prism.

Statistical Analysis. Data are expressed as means \pm S.D. Statistical analyses were performed with two-tailed Student's $t$ test (for two groups) or two-way ANOVA (for multiple groups). $P<0.05$ was considered as statistically significant.

\section{Results}

Systemic Pharmacokinetics of DTG in WT and Abcg2-Null Mice. Abcg2-null mice were confirmed by PCR genotyping (Fig. 2A). In addition, we used PPIX, a well-known substrate of ABCG2 (Jonker et al., 2002, 2007), to validate the function of ABCG2 in Abcg2-null mice. As expected, PPIX level was statistically significantly increased in the liver of Abcg2-null mice when compared with WT mice (Fig. 2B), indicating that ABCG2 is functionally deficient in Abcg2-null mice. After DTG treatment, it was quickly absorbed in both WT and Abcg2-null mice (Fig. 2C). Because DTG is a substrate of ABCG2 (Reese et al., 2013), we hypothesized that deficiency of ABCG2 slows down DTG excretion and leads to its accumulation in the body. However, no statistically significant difference was found in the area under serum DTG concentration-time curve $\left(\mathrm{AUC}_{0-48}\right)$ of $\mathrm{WT}$ and Abcg2-null mice (Fig. 2, C and D). In addition, no genotype-related differences were found in other pharmacokinetic parameters including clearance, elimination half-life, or volume of distribution (Fig. 2, E-G). These results indicate that deficiency of ABCG2 does not statistically significantly alter the systemic pharmacokinetics of DTG.

Tissue-Specific Pharmacokinetics of DTG in WT and Abcg2-Null Mice. Because ABCG2 is expressed in the liver, kidney, and brain (Maliepaard et al., 2001; Fetsch et al., 2006), we examined DTG levels in these organs of Abcg2-null mice. After DTG treatment, high levels of DTG were observed in the liver and kidney of both WT and Abcg2-null mice (Fig. 3, A-D). The levels of DTG in the brain were much lower than those in the liver and kidney (Fig. 3, E and F). However, no genotype-related difference was found in DTG pharmacokinetics in the liver, kidney, or brain of WT and Abcg2-null mice (Fig. 3). These data indicate that ABCG2 is not critical
A

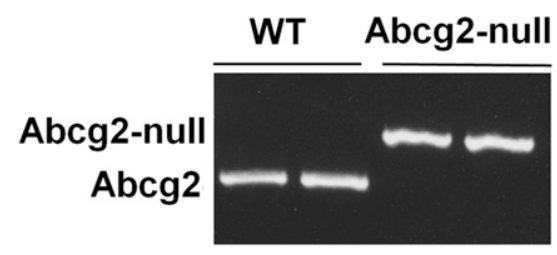

C
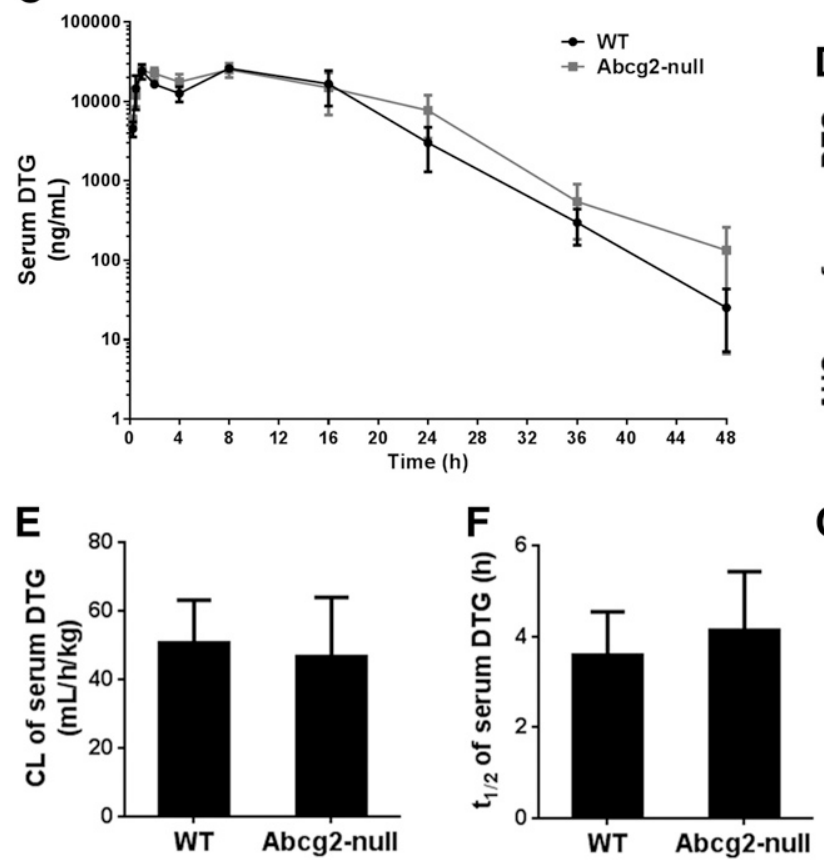

G

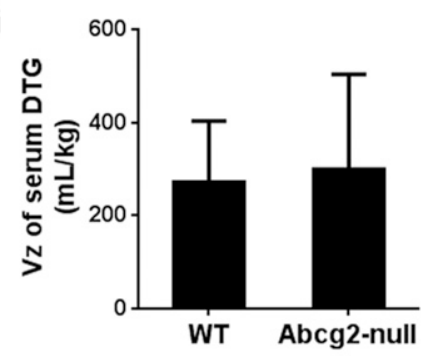

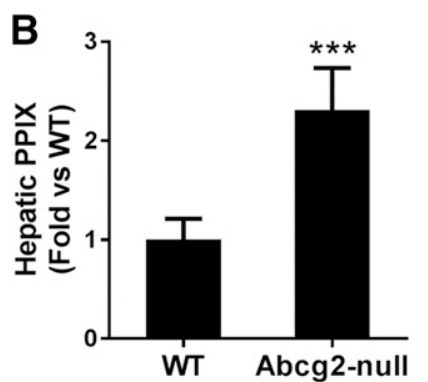

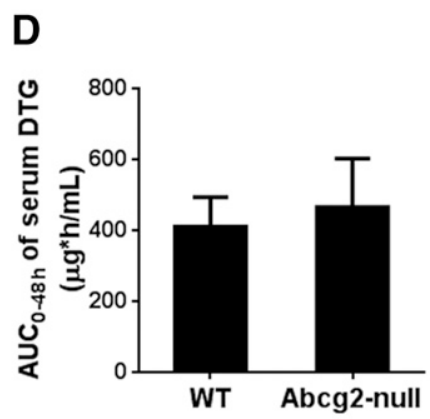

Fig. 2. Systemic pharmacokinetics of DTG in WT and Abcg2-null mice. (A and B) Genetic and functional validation of Abcg2-null mice. PPIX was used as a substrate of ABCG2. (C) Serum concentrationtime curves of DTG in WT and Abcg2-null mice. The mice were administrated with a single dose of DTG (20 mg/kg, p.o.). (D-G) Comparison of major pharmacokinetic parameters of DTG between WT and Abcg2-null mice. PPIX and DTG were measured by UPLC-QTOFMS. Pharmacokinetic parameters were calculated by noncompartmental model analysis on Phoenix WinNonlin. All data are expressed as means \pm S.D. $(n=4)$. $* * * P<0.001 \mathrm{vs}$. WT. CL, clearance; $t_{1 / 2}$, elimination half-life; $\mathrm{V}_{\mathrm{Z}}$, volume of distribution. 


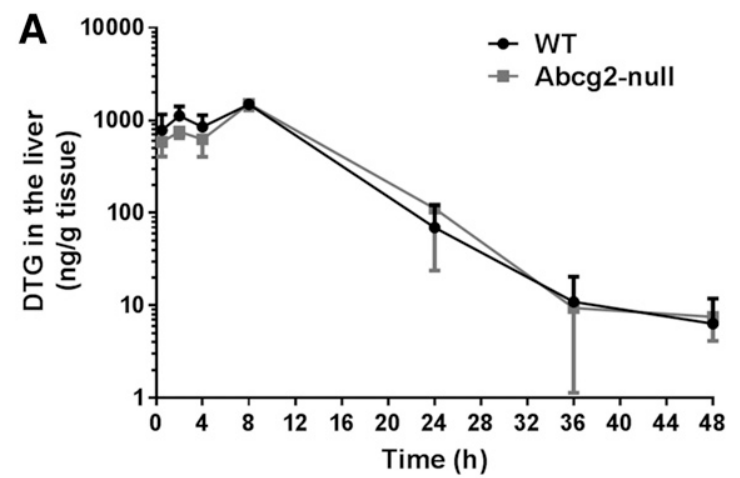

B
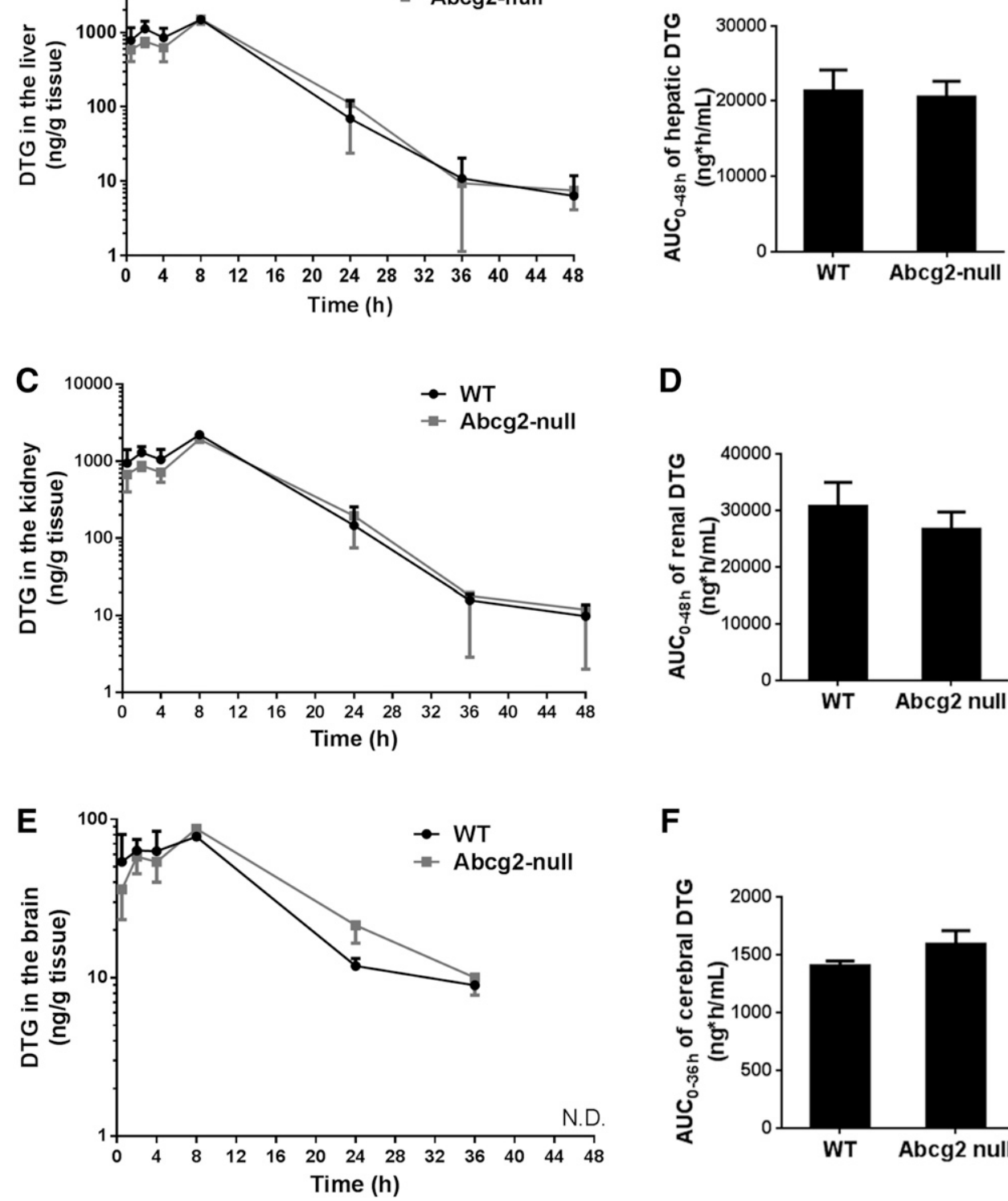

D

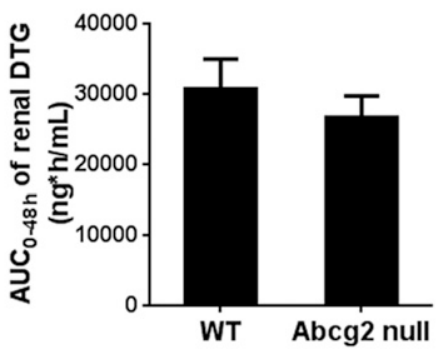

Fig. 3. Tissue-specific pharmacokinetics of DTG in WT and Abcg2-null mice. The mice were administrated with a single dose of DTG (20 mg/kg, p.o.). (A, C, and E) Concentration-time curves of DTG in the liver, kidney, and brain of WT and Abcg2-null mice. (B, D, and F) AUCs of DTG in the liver, kidney, and brain of WT and Abcg2-null mice. AUCs were calculated by Graphpad Prism. All data are expressed as means \pm S.D. $(n=4)$. N.D., not detected.

$\mathbf{F}$

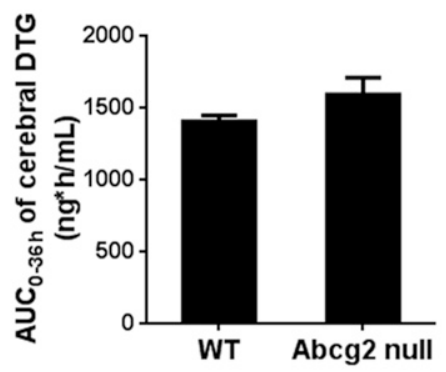

in modulating DTG excretion from the liver, kidney, and brain.

Excretion of DTG in WT and Abcg2-Null Mice. In humans, DTG is mainly excreted through feces (Castellino et al., 2013). We observed a similar pattern of DTG excretion in mice, as a high level of DTG was found in feces but not in urine (Fig. 4, A and B). If ABCG2 is important in DTG excretion, DTG level should decrease in feces and may increase in urine of Abcg2-null mice. However, DTG level in feces of Abcg2-null mice did not decrease when compared with WT mice, and it actually slightly increased (Fig. 4A). In addition, no statistically significant differences in DTG levels were observed in the urine of WT and Abcg2-null mice (Fig. 4B). These data suggest that ABCG2 deficiency does not alter DTG excretion.

Metabolism of DTG in WT and Abcg2-Null Mice. DTG metabolites were identified in both feces and urine, although their abundances were much lower than the parent drug (Fig. 4, C-F). We hypothesized that deficiency of ABCG2 will retain DTG in the liver of Abcg2-null mice, which will increase DTG metabolism because DTG metabolizing enzymes, including CYP3A and UGT, are highly expressed in the liver (Guengerich, 1999; Nakamura et al., 2008). However, no statistically significant increase was found among the four major metabolites of DTG (M1, M2, M3, and M4) in Abcg2-null mice (Fig. 4, G and H). These data indicate that ABCG2 deficiency does not statistically significantly alter DTG metabolism.

\section{Discussion}

Abcg2-null mice have been used to explore the impact of ABCG2 deficiency on drug metabolism and pharmacokinetics. Both pitavastatin and nitrofurantoin are ABCG2 substrates, and their excretion was statistically significantly decreased in Abcg2-null mice (Hirano et al., 2005; Merino et al., 2005). The plasma AUC of sunitinib, a substrate of ABCG2, was $\sim$ four fold higher in Abcg2-null mice than that in WT mice, 
A

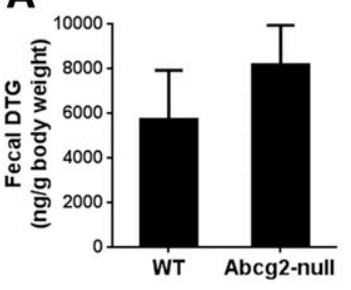

B

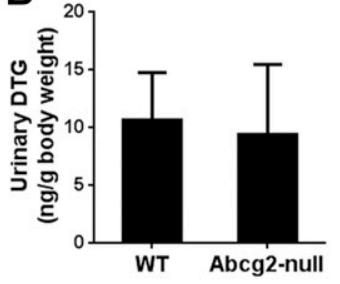

C $\mathrm{WT}_{\mathrm{T}}$ feces

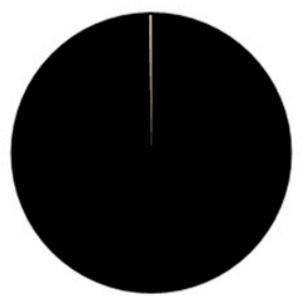

D Abcg2-null, feces

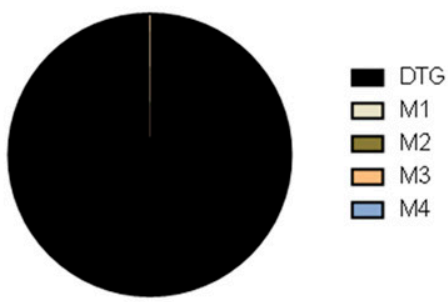

E

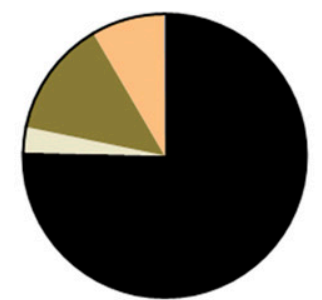

F Abcg2-null, urine

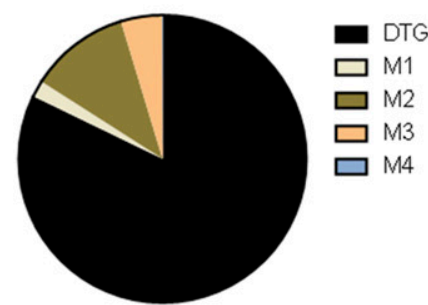

G

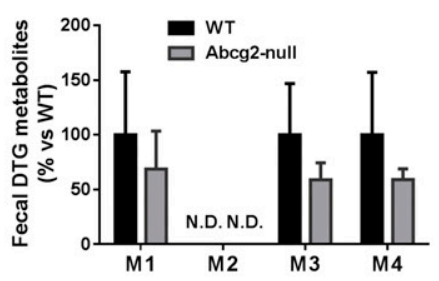

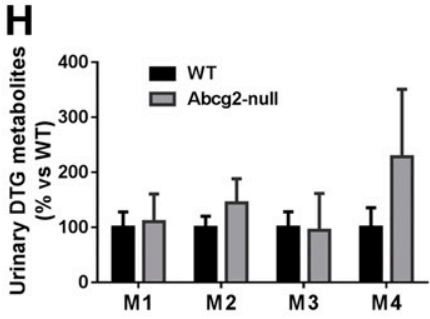

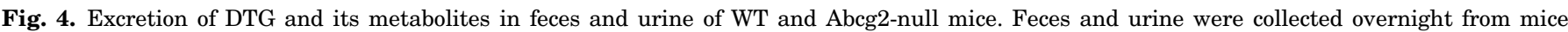

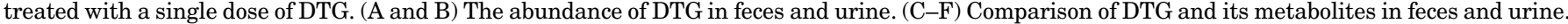

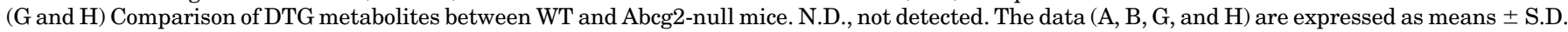
$(n=4)$.

consistent with human data showing high AUCs in individuals with ABCG2 deficiency (Mizuno et al., 2012). These data demonstrate that the Abcg2-null mouse model is an ideal tool to determine the impact of ABCG2 deficiency on drug metabolism and pharmacokinetics. The current work used Abcg2-null mice to investigate the role of ABCG2 in DTG metabolism and pharmacokinetics. We found that ABCG2 deficiency had no statistically significant impact on DTG metabolism and pharmacokinetics.

Because DTG is a substrate of ABCG2 (Reese et al., 2013), we expected a high AUC of DTG in Abcg2-null mice when compared with WT mice. However, no genotype-related difference was found in either systemic or tissue-specific AUCs of DTG, indicating that ABCG2 is not critical in modulating DTG distribution and disposition. In addition, we found that ABCG2 deficiency had no statistically significant impact on DTG metabolism and excretion. Our data did not support the finding in a previous report showing high plasma concentrations of DTG in ABCG2deficient individuals (Tsuchiya et al., 2017). In this clinical report, the number of ABCG2-deficient individuals with SNP rs2231142 homozygous is three, which might be too small to evaluate group differences. In addition, this clinical study only used a limited time window to monitor DTG concentrations instead of a full pharmacokinetic study. Furthermore, plasma concentrations of DTG in individuals with SNP rs2231142 heterozygous genotype $(n=17)$ did not increase when compared with WT genotype $(n=22)$ (Tsuchiya et al., 2017), suggesting that ABCG2 is not critical in DTG excretion.

Our data are supported by a previous report showing that concomitant use of DTG with lopinavir and ritonavir, two potent inhibitors of ABCG2 and ABCB1 (Kis et al., 2010), did not cause statistically significant changes in DTG pharmacokinetics (Song et al., 2011). Interestingly, coadministration of DTG with darunavir and ritonavir leads to a statistically significant reduction in DTG exposure (Song et al., 2011), which suggests that DTG may be a substrate of certain uptake transporter(s), such as organic anion transporting polypeptide transporters, as darunavir is an inhibitor of organic anion transporting polypeptide transporters (Kis et al., 2010). Further studies are needed to determine the specific transporters in DTG uptake and efflux.

In summary, the current work revealed that ABCG2 deficiency did not alter DTG metabolism and pharmacokinetics, suggesting that dose adjustment of DTG is not needed for individuals with ABCG2 deficiency.

\section{Authorship Contributions}

Participated in research design: Zhu, McMahon, Ma.

Conducted experiments: Zhu, Tian.

Contributed new reagents or analytic tools: Zhu, Ma.

Performed data analysis: Zhu, Tian, Shehu, Ma.

Wrote or contributed to the writing and editing of the manuscript: Zhu, McMahon, Ma.

\section{References}

Bennetto-Hood C, Tabolt G, Savina P, and Acosta EP (2014) A sensitive HPLC-MS/MS method for the determination of dolutegravir in human plasma. $J$ Chromatogr $B$ Analyt Technol Biomed Life Sci 945-946:225-232.

Cahn P, Pozniak AL, Mingrone H, Shuldyakov A, Brites C, Andrade-Villanueva JF, Richmond G, Buendia CB, Fourie J, Ramgopal M, et al.; extended SAILING Study Team (2013) Dolutegravir versus raltegravir in antiretroviral-experienced, integrase-inhibitor-naive adults with HIV: week 48 results from the randomised, double-blind, non-inferiority SAILING study. Lancet 382:700-708.

Castellino S, Moss L, Wagner D, Borland J, Song I, Chen S, Lou Y, Min SS, Goljer I, Culp A, et al. (2013) Metabolism, excretion, and mass balance of the HIV-1 integrase inhibitor dolutegravir in humans. Antimicrob Agents Chemother 57: 3536-3546.

de Boer MG, van den Berk GE, van Holten N, Oryszcyn JE, Dorama W, Moha DA, and Brinkman K (2016) Intolerance of dolutegravir-containing combination antiretroviral therapy regimens in real-life clinical practice. AIDS 30:2831-2834.

Eisenblätter T, Hüwel S, and Galla H-J (2003) Characterisation of the brain multidrug resistance protein (BMDP/ABCG2/BCRP) expressed at the blood-brain barrier. Brain Res 971:221-231.

Elzi L, Erb S, Furrer H, Cavassini M, Calmy A, Vernazza P, Günthard H, Bernasconi E, and Battegay M; Swiss HIV Cohort Study Group (2017) Adverse events of raltegravir and dolutegravir. AIDS 31:1853-1858.

Eron JJ, Clotet B, Durant J, Katlama C, Kumar P, Lazzarin A, Poizot-Martin I, Richmond G, Soriano V, Ait-Khaled M, et al.; VIKING Study Group (2013) Safety and efficacy of dolutegravir in treatment-experienced subjects with raltegravirresistant HIV type 1 infection: 24 -week results of the VIKING Study. J Infect Dis 207:740-748. 
Fetsch PA, Abati A, Litman T, Morisaki K, Honjo Y, Mittal K, and Bates SE (2006) Localization of the ABCG2 mitoxantrone resistance-associated protein in normal tissues. Cancer Lett 235:84-92.

Guengerich FP (1999) Cytochrome P-450 3A4: regulation and role in drug metabolism. Annu Rev Pharmacol Toxicol 39:1-17.

Hightower KE, Wang R, Deanda F, Johns BA, Weaver K, Shen Y, Tomberlin GH, Carter HL III, Broderick T, Sigethy S, et al. (2011) Dolutegravir (S/GSK1349572) exhibits significantly slower dissociation than raltegravir and elvitegravir from wild-type and integrase inhibitor-resistant HIV-1 integrase-DNA complexes. Antimicrob Agents Chemother 55:4552-4559.

Hira D and Terada T (2018) BCRP/ABCG2 and high-alert medications: biochemical, pharmacokinetic, pharmacogenetic, and clinical implications. Biochem Pharmaco 147:201-210.

Hirano M, Maeda K, Matsushima S, Nozaki Y, Kusuhara H, and Sugiyama Y (2005) Involvement of BCRP (ABCG2) in the biliary excretion of pitavastatin. Mol Pharmacol 68:800-807.

Hoffmann C, Welz T, Sabranski M, Kolb M, Wolf E, Stellbrink HJ, and Wyen C (2017) Higher rates of neuropsychiatric adverse events leading to dolutegravir discontinuation in women and older patients. HIV Med 18:56-63.

Iida A, Saito S, Sekine A, Mishima C, Kitamura Y, Kondo K, Harigae S, Osawa S, and Nakamura Y (2002) Catalog of 605 single-nucleotide polymorphisms (SNPs) among 13 genes encoding human ATP-binding cassette transporters: ABCA4, ABCA7, ABCA8, ABCD1, ABCD3, ABCD4, ABCE1, ABCF1, ABCG1, ABCG2, ABCG4, ABCG5, and ABCG8. J Hum Genet 47:285-310.

Imai Y, Nakane M, Kage K, Tsukahara S, Ishikawa E, Tsuruo T, Miki Y, and Sugimoto Y (2002) C421A polymorphism in the human breast cancer resistance protein gene is associated with low expression of Q141K protein and low-level drug resistance. Mol Cancer Ther 1:611-616.

Jonker JW, Buitelaar M, Wagenaar E, Van Der Valk MA, Scheffer GL, Scheper RJ, Plosch T, Kuipers F, Elferink RP, Rosing H, et al. (2002) The breast cancer resistance protein protects against a major chlorophyll-derived dietary phototoxin and protoporphyria. Proc Natl Acad Sci USA 99:15649-15654.

Jonker JW, Musters S, Vlaming MLH, Plösch T, Gooijert KER, Hillebrand MJ, Rosing H, Beijnen JH, Verkade HJ, and Schinkel AH (2007) Breast cancer resistance protein (Bcrp1/Abcg2) is expressed in the harderian gland and mediates transport of conjugated protoporphyrin IX. Am J Physiol Cell Physiol 292 C2204-C2212.

Keskitalo JE, Zolk O, Fromm MF, Kurkinen KJ, Neuvonen PJ, and Niemi M (2009) ABCG2 polymorphism markedly affects the pharmacokinetics of atorvastatin and rosuvastatin. Clin Pharmacol Ther 86:197-203.

Kis O, Robillard K, Chan GN, and Bendayan R (2010) The complexities of antiretroviral drug-drug interactions: role of $\mathrm{ABC}$ and SLC transporters. Trends Pharmacol Sci 31:22-35.

Kobayashi M, Yoshinaga T, Seki T, Wakasa-Morimoto C, Brown KW, Ferris R, Foster SA, Hazen RJ, Miki S, Suyama-Kagitani A, et al. (2011) In vitro antiretroviral properties of S/GSK1349572, a next-generation HIV integrase inhibitor. Antimicrob Agents Chemother 55:813-821.

Liu K, Zhu J, Huang Y, Li C, Lu J, Sachar M, Li S, and Ma X (2017) Metabolism of KO143, an ABCG2 inhibitor. Drug Metab Pharmacokinet 32:193-200.

Maliepaard M, Scheffer GL, Faneyte IF, van Gastelen MA, Pijnenborg AC, Schinkel AH, van De Vijver MJ, Scheper RJ, and Schellens JH (2001) Subcellular localization and distribution of the breast cancer resistance protein transporter in normal human tissues. Cancer Res 61:3458-3464.

Merino G, Jonker JW, Wagenaar E, van Herwaarden AE, and Schinkel AH (2005) The breast cancer resistance protein (BCRP/ABCG2) affects pharmacokinetics, hepatobiliary excretion, and milk secretion of the antibiotic nitrofurantoin. Mol Pharmacol 67:1758-1764.

Min S, Song I, Borland J, Chen S, Lou Y, Fujiwara T, and Piscitelli SC (2010) Pharmacokinetics and safety of S/GSK1349572, a next-generation HIV integrase inhibitor, in healthy volunteers. Antimicrob Agents Chemother 54:254-258.
Mizuno T, Fukudo M, Terada T, Kamba T, Nakamura E, Ogawa O, Inui K, and Katsura T (2012) Impact of genetic variation in breast cancer resistance protein (BCRP/ABCG2) on sunitinib pharmacokinetics. Drug Metab Pharmacokinet 27:631-639.

Nakamura A, Nakajima M, Yamanaka H, Fujiwara R, and Yokoi T (2008) Expression of UGT1A and UGT2B mRNA in human normal tissues and various cell lines. Drug Metab Dispos 36:1461-1464.

Nicolazzo JA and Katneni K (2009) Drug transport across the blood-brain barrier and the impact of breast cancer resistance protein (ABCG2). Curr Top Med Chem $\mathbf{9}$ : 130-147.

Parant F, Miailhes P, Brunel F, and Gagnieu MC (2018) Dolutegravir-related neurological adverse events: a case report of successful management with therapeutic drug monitoring. Curr Drug Saf 13:69-71.

Phillips AN, Cambiano V, Nakagawa F, Revill P, Jordan MR, Hallett TB, Doherty M, De Luca A, Lundgren JD, Mhangara M, et al.; Working Group on Modelling Potential Responses to High Levels of Pre-ART Drug Resistance in Sub-Saharan Africa (2018) Cost-effectiveness of public-health policy options in the presence of pretreatment NNRTI drug resistance in sub-Saharan Africa: a modelling study. Lancet HIV 5:e146-e154.

Raffi F, Rachlis A, Stellbrink H-J, Hardy WD, Torti C, Orkin C, Bloch M, Podzamczer D, Pokrovsky V, Pulido F, et al.; SPRING-2 Study Group (2013) Once-daily dolutegravir versus raltegravir in antiretroviral-naive adults with HIV-1 infection: 48 week results from the randomised, double-blind, non-inferiority SPRING-2 study. Lancet 381:735-743.

Reese M.J, Savina PM, Generaux GT, Tracey H, Humphreys JE, Kanaoka E, Webster LO, Harmon KA, Clarke JD, and Polli JW (2013) In vitro investigations into the roles of drug transporters and metabolizing enzymes in the disposition and drug interactions of dolutegravir, a HIV integrase inhibitor. Drug Metab Dispos 41: 353-361.

Song I, Min SS, Borland J, Lou Y, Chen S, Patel P, Ishibashi T, and Piscitelli SC (2011) The effect of lopinavir/ritonavir and darunavir/ritonavir on the HIV integrase inhibitor S/GSK1349572 in healthy participants. J Clin Pharmacol 51:237-242.

Tsuchiya K, Hayashida T, Hamada A, Oki S, Oka S, and Gatanaga H (2017) High plasma concentrations of dolutegravir in patients with ABCG2 genetic variants. Pharmacogenet Genomics 27:416-419.

Wan Z, Wang G, Li T, Xu B, Pei Q, Peng Y, Sun H, Cheng L, Zeng Y, Yang G, et al. (2015) Marked alteration of rosuvastatin pharmacokinetics in healthy Chinese with ABCG2 $34 \mathrm{G}>\mathrm{A}$ and $421 \mathrm{C}>\mathrm{A}$ homozygote or compound heterozygote. $J$ Pharmacol Exp Ther 354:310-315.

Wang P, Sachar M, Lu J, Shehu AI, Zhu J, Chen J, Liu K, Anderson KE, Xie W, Gonzalez FJ, et al. (2019) The essential role of the transporter ABCG2 in the pathophysiology of erythropoietic protoporphyria. Sci Adv 5:eaaw6127.

Yagura $\mathrm{H}$, Watanabe D, Kushida $\mathrm{H}$, Tomishima K, Togami H, Hirano A, Takahashi M, Hirota K, Ikuma M, Kasai D, et al. (2017) Impact of UGT1A1 gene polymorphisms on plasma dolutegravir trough concentrations and neuropsychiatric adverse events in Japanese individuals infected with HIV-1. BMC Infect Dis 17 $622-629$.

Zhu J, Wang P, Li F, Lu J, Shehu AI, Xie W, McMahon D, and Ma X (2018a) CYP1A1 and 1B1-mediated metabolic pathways of dolutegravir, an HIV integrase inhibitor. Biochem Pharmacol 158:174-184.

Zhu J, Wang P, Shehu AI, Lu J, Bi H, and Ma X (2018b) Identification of novel pathways in idelalisib metabolism and bioactivation. Chem Res Toxicol 31: $548-555$.

Address correspondence to: Dr. Xiaochao Ma, Center for Pharmacogenetics, Department of Pharmaceutical Sciences, School of Pharmacy, University of Pittsburgh, 335 Sutherland Drive, Pittsburgh, PA 15261. E-mail: mxiaocha@pitt.edu 